\title{
Correction to: Comparing methods to estimate incremental inpatient costs and length of stay due to methicillin-resistant Staphylococcus aureus in Alberta, Canada
}

Erin Kirwin ${ }^{1 *}$, Marie Varughese ${ }^{1}$, David Waldner ${ }^{2}$, Kimberley Simmonds ${ }^{1,3,4}$, A. Mark Joffe ${ }^{2,5}$ and Stephanie Smith ${ }^{2}$

Correction to: BMC Health Serv Res (2019) 19:743

https://doi.org/10.1186/s12913-019-4578-z

In the original publication of this article [1], the authors want to add the following sentence in the Acknowledgement section:

The authors thank the Infection Prevention and Control (IPC) Program at Alberta Health Services for providing access to the IPC surveillance platform data.

\footnotetext{
Author details

${ }^{1}$ Alberta Ministry of Health, Edmonton, Alberta, Canada. ${ }^{2}$ Department of Medicine, University of Alberta, Edmonton, Alberta, Canada. ${ }^{3}$ School of Public Health, University of Alberta, Edmonton, Alberta, Canada. ${ }^{4}$ Department of Community Health Sciences, University of Calgary, Calgary, Alberta, Canada. ${ }^{5}$ Alberta Health Services, Edmonton, Alberta, Canada.
}

Published online: 31 December 2019

\footnotetext{
Reference

1. Kirwin E, et al. Comparing methods to estimate incremental inpatient costs and length of stay due to methicillin-resistant Staphylococcus aureus in Alberta, Canada. BMC Health Serv Res. 2019;19:743. https://doi.org/10.1186/ s12913-019-4578-z.
}

Full list of author information is available at the end of the article 